\title{
Looking for cosmic neutrino background
}

\author{
Chiaki Yanagisawa ${ }^{1,2 *}$ \\ ${ }^{1}$ Department of Physics and Astronomy, Stony Brook University, Stony Brook, NY, USA \\ ${ }^{2}$ Science Department, Borough of Manhattan Community College, City University of New York, New York, NY, USA
}

\section{Edited by:}

Omar G. Miranda, Centro de

Investigacion y de Estudios

Avanzados del IPN, Mexico

Reviewed by:

Bhupal Dev, University of

Manchester, UK

Alexis Aguilar-Arevalo, Universidad

Nacional Autónoma de México,

Mexico

\section{*Correspondence:} Physics and Astronomy, Stony Brook University, NY 11794-3800, USA

e-mail:chiaki@

nngroup.physics.sunysb.edu
Chiaki Yanagisawa, Department of

Since the discovery of neutrino oscillation in atmospheric neutrinos by the Super-Kamiokande experiment in 1998, study of neutrinos has been one of exciting fields in high-energy physics. All the mixing angles were measured. Quests for (1) measurements of the remaining parameters, the lightest neutrino mass, the $\mathrm{CP}$ violating phase(s), and the sign of mass splitting between the mass eigenstates $m_{3}$ and $m_{1}$, and (2) better measurements to determine whether the mixing angle $\theta_{23}$ is less than $\pi / 4$, are in progress in a well-controlled manner. Determining the nature of neutrinos, whether they are Dirac or Majorana particles is also in progress with continuous improvement. On the other hand, although the ideas of detecting cosmic neutrino background have been discussed since 1960s, there has not been a serious concerted effort to achieve this goal. One of the reasons is that it is extremely difficult to detect such low energy neutrinos from the Big Bang. While there has been tremendous accumulation of information on Cosmic Microwave Background since its discovery in 1965, there is no direct evidence for Cosmic Neutrino Background. The importance of detecting Cosmic Neutrino Background is that, although detailed studies of Big Bang Nucleosynthesis and Cosmic Microwave Background give information of the early Universe at $\sim a$ few minutes old and $\sim 300 \mathrm{k}$ years old, respectively, observation of Cosmic Neutrino Background allows us to study the early Universe at $\sim 1 \mathrm{~s}$ old. This article reviews progress made in the past 50 years on detection methods of Cosmic Neutrino Background.

Keywords: big-bang cosmology, cosmic neutrino background, cosmic-rays, neutrino elastic-scattering, neutrino capture by beta-decaying nuclei

\section{INTRODUCTION}

According to the Big Bang theory, neutrinos decoupled from other particles earlier than Cosmic Microwave Background (CMB). Detecting these neutrinos will give direct information of the earliest possible epoch of the Universe we can observe after the Big Bang. This review starts first with a brief summary of Cosmic Neutrino Background $(\mathrm{C} v \mathrm{~B})$ according to the Big Bang theory with the Standard Model of particle physics except that, as observed, neutrinos are massive rather than massless fundamental particles. There have been ideas on how to detect $\mathrm{C} v \mathrm{~B}$ since 1960s. These proposed detection methods can be classified into two types: direct detection and indirect detection method. The direct detection methods can further be divided into two classes: measurements of energy/momentum transfer from $\mathrm{C} v \mathrm{~B}$ neutrinos to target materials in terms of acceleration of the targets through torsion unbalance or possibly through calorimetry, and measurements of energy of outgoing electrons produced by neutrino capture by $\beta$-decaying nuclei. The indirect method basically measures the spectrum of ultra-high energy cosmic rays and identify threshold effects/dips due to interactions between ultra-high energy neutrinos or other cosmic rays from some sources and neutrinos from $\mathrm{C} v \mathrm{~B}$.

\section{THE STANDARD BIG BANG COSMOLOGY AND ITS PREDICTIONS}

According to the standard Big Bang cosmology [1-3] the number density of a relativistic particle of type $i$ of momentum $\vec{p}$, the energy $E_{i}$ and the internal degree of freedom $g_{i}$ ( 2 for $\gamma, 1$ for $v$ or $\bar{v})$ at temperature $T$ is given by

$$
n_{i}=\frac{g_{i}}{(2 \pi)^{3}} \int f_{i}(\vec{p}) \mathrm{d}^{3} p \text { and } \rho_{i}=\frac{g_{i}}{(2 \pi)^{3}} \int E_{i}(\vec{p}) f_{i}(\vec{p}) \mathrm{d}^{3} p
$$

where $\left.f_{i}(\vec{p})=1 /\left[\exp \left(\left(E_{i}-\mu_{i}\right) / T\right) \pm 1\right)\right]$ with chemical potential $\mu_{i}$ ( + for fermion and - for boson). From Equation (1) for a relativistic boson of type $i$ its number density is $\zeta(3) g_{i} T^{3} / \pi^{2}$ and for a relativistic fermion $(3 / 4) \zeta(3) g_{i} T^{3} / \pi^{2}$ where $\zeta$ is the Riemann zeta function. Also the energy density is $\left(\pi^{2} / 30\right) g_{i} T^{4}$ for a boson and $(7 / 8)\left(\pi^{2} / 30\right) g_{i} T^{4}$ for a fermion [3].

The time dependence of the scale factor $a(t)$ of the Universe, when radiation is dominant, is expressed by $a(t) \sim t^{1 / 2}$. By definition the Hubble parameter is the ratio $\dot{a} / a$ and thus $H=$ $\dot{a} / a=1 / 2 t$. From the Friedman equation for radiation-dominant epoch, the Hubble parameter is

$$
H^{2}=\frac{8 \pi G}{3} \rho_{R}=\frac{8 \pi G}{3} \frac{\pi^{2}}{30} g_{e f f} T^{4}=2.76 \frac{g_{e f f} T^{4}}{M_{P l}^{2}},
$$

where $G$ is the universal gravitational constant, $\rho_{R}$ is the energy density of radiation, $M_{P l}$ is the Planck mass, and $g_{e f f}$ is the total number of effective degrees of freedom of relativistic particles in thermal equilibrium at temperature $T$ which is given by 


$$
g_{\text {eff }}=\sum_{i=\text { bosons }} g_{i}+\frac{7}{8} \sum_{j=\text { fermions }} g_{j} .
$$

Combining Equations $(2,3)$, the relation between the time $t$ and the temperature $T$ can be found as

$$
t=0.30 \frac{M_{P l}}{\sqrt{g_{e f f}} T^{2}} \sim\left(\frac{1 M e V}{T}\right)^{2} \mathrm{sec}
$$

When the interaction rate of neutrino $\Gamma_{v}$ becomes smaller than the expansion rate of the Universe i.e., $H \sim T^{2} / M_{P l}$, neutrinos decouple from other particles and stop interacting with them at the decoupling temperature $T_{v, \operatorname{dec}} . \Gamma_{v}$ is $\sigma_{v} v_{v} n_{v}$ where $\sigma_{v} \approx$ $\alpha^{2} T^{2} / m_{W}^{4}$ is the typical neutrino cross section with the fine structure constant $\alpha$, W mass $m_{W}, v_{v}$ neutrino velocity $\sim c=1$, and the neutrino number density $n_{v} \propto T^{3} . \Gamma_{v}$ is then $\sim \alpha^{2} T^{5} / m_{W}^{4}$. When $\Gamma / H \sim \alpha^{2} M_{P l} T^{3} / m_{W}^{4}=1, T$ corresponds to the neutrino decoupling temperature $T_{v, \text { dec }} \sim\left(m_{W}^{4} / \alpha^{2} M_{P l}\right)^{1 / 3} \sim 4 \mathrm{MeV}$ [3]. More detailed calculations show that $T_{\nu, d e c}=2-3 \mathrm{MeV}$ for $\nu_{e}$ and $3.5 \mathrm{MeV}$ for $v_{\mu, \tau}$. From Equation (4) the neutrino decoupling occurs at $\sim 0.3-1.0 \mathrm{~s}$ after the Big Bang. While $\mathrm{CMB}$ provides the information of the Universe at 300,000 years after the Big Bang, the study of nucleosynthesis gives the information of the Universe at 200-1000s after the Big Bang [3,4]. Observation of $\mathrm{C} v \mathrm{~B}$, therefore, would provide the information of the earliest observable Universe.

\subsection{COSMIC NEUTRINO BACKGROUND}

As described in the previous section neutrinos decouple with electrons, positrons and photons at temperature $T \sim$ a few $\mathrm{MeV}$ and remain as such until today. Soon after the neutrino decoupling, at about $T=0.5 \mathrm{MeV}, e^{ \pm}$pair annihilate into photons and transfer their entropy to these photons. This transfer of entropy to photons effectively slows down the rate of decrease in photon temperature compared with that in neutrino temperature as the Universe expands [3]. Since the entropy of neutrinos is conserved, $T_{v}=(4 / 11)^{1 / 3} T_{\gamma}$ and, since $T_{\gamma}^{\text {now }}=2.7 \mathrm{~K}, T_{v}^{\text {now }}=1.9 \mathrm{~K}=$ $1.7 \times 10^{-4} \mathrm{eV}$. Therefore the number density of neutrinos is given by $n_{v}=(3 / 22) n_{\gamma}=56 \mathrm{~cm}^{-3}$ per flavor. In the standard Big Bang model it is assumed that $n_{v}=n_{\bar{v}}$ and neutrinos' chemical potentials are zero. However, in this review it is not assumed, in which case it is convenient to introduce an asymmetry parameter for neutrino with flavor $\alpha, v_{\alpha}: \eta_{\alpha}=\left(n_{v_{\alpha}}-n_{\bar{v}_{\alpha}}\right) / n_{\gamma}=$ $\left[\pi^{2} / 12 \zeta(3)\right]\left(\xi_{\alpha}+\xi_{\alpha}^{3} / \pi^{2}\right)\left(T_{v} / T_{\gamma}\right)^{3}$ where $\xi_{\alpha}=\mu_{\alpha} / T$ with $\mu_{\alpha}$ being the chemical potential of $v_{\alpha}$ [5]. A recent study of the primordial He abundance data and WMAP data finds that the sum of the asymmetries of all neutrino flavors, taking into account the effect of neutrino oscillation with the neutrino mixing angle $\sin ^{2} \theta_{13}=0.04(0.00)$, is $-0.071(-0.064) \leq \eta_{v} \leq 0.054(0.072)$ (90\% C.L.) [6]. At the time of neutrino decoupling $\left(T_{v, d e c} \sim \mathrm{a}\right.$ few $\mathrm{MeV}$ ), since we know that neutrino masses are much less than a few $\mathrm{MeV}$, all neutrinos are relativistic ( for the status of neutrino masses refer to the review by the Particle Data Group) [7]. However, as the current neutrino temperature is $1.9 \mathrm{~K}(1.7 \times$ $10^{-4} \mathrm{eV}$ ), some of neutrinos may be non-relativistic, in which case whether neutrinos are Dirac or Majorana type is important.
From a recent analysis by the Planck collaboration using the $\mathrm{CMB}$ and Baryon Acoustic Oscillation (BAO) data, the effective number of relativistic active neutrinos at the time of decoupling of the CMB $N_{\text {eff }}$ is found to be $3.30_{-0.51}^{+0.51}$, consistent with the existence of three active neutrinos [8]. However, according to the analysis using the Planck, WMAP and BICEP2 data by Giusarma et al., $N_{\text {eff }}$ is found to be $4.00 \pm 0.41$ [9].

\subsection{CLUSTERING OF C $v$ B}

As we know that neutrino has mass, it is possible that $\mathrm{C} v \mathrm{~B}$ can be trapped in gravitational potential well of some structures in the Universe such as large galaxies and clusters of galaxies when $\mathrm{C} v \mathrm{~B}$ has velocity smaller than the escape velocity. For a large galaxy like Milky Way or a large cluster of galaxies the escape velocities are $\sim 600$ or $\sim 2000 \mathrm{~km} / \mathrm{s}$, respectively. From the Maxwell-Boltzmann distribution, the average velocity of non-relativistic neutrino of mass $m_{v}$ at temperature $T_{v},<$ $\left|\beta_{v}\right|>=\sqrt{8 k T_{v} /\left(\pi m_{v}\right)}=\sqrt{4.3 \times 10^{-4} \mathrm{eV} / m_{v}}$. Therefore < $\left|v_{v}\right|>=19,600(6200) \mathrm{km} / \mathrm{s}$ for $m_{v}=0.1(1.0) \mathrm{eV}$ [10]. It seems that only a small fraction of neutrinos can be gravitationally trapped in a large cluster of galaxies. However, detailed simulation that takes into account either the current static mass distribution of the Milky Way (MWnow) or an estimated halo mass distribution before the formation of Milky Way through baryonic compression (NFWhalo) reveals that there may be local neutrino overdensity effect at the position of our solar system [11]. The simulation consists of several types of weakly interacting, selfgravitating particles (cold dark matter and neutrinos) modeled as a multi-component collisionless gas with the Vlasov equation and uses the density profile of cold dark matter (CDM) proposed by Navarro, Frank and White (NFWhalo) [12]. The neutrino overdensity $\left.n_{v} /<n_{v}\right\rangle$, where $\left\langle n_{v}\right\rangle$ is the average neutrino density predicted by the standard Big Bang model, is with the NFWhalo model, 12 and 1.4 for $m_{v}=0.6$ and $0.15 \mathrm{eV}$, respectively, and with the MWnow model, 20 and 1.6 for $m_{v}=$ $0.6 \mathrm{eV}$ and $0.15 \mathrm{eV}$, respectively. In the both cases (NFWhalo and MWnow) when $m_{v}<0.1 \mathrm{eV}$, there is no overdensity. Overdensity values 20 and 1.4 correspond to values for the neutrino asymmetry parameter $\eta_{v}, 2.7$ and 0.19 , respectively [11].

\section{CvB DETECTION METHODS}

Several methods to detect $\mathrm{C} v \mathrm{~B}$ have been proposed and these proposed methods can be divided into three categories: (1) direct detection of coherent $\mathrm{C} v \mathrm{~B}$ elastic scattering with target nuclei through momentum transfer, (2) direct detection by neutrino capture by $\beta$-decaying nuclei, and (3) indirect method by finding spectral distortion through $\mathrm{C} v \mathrm{~B}$ interaction with ultra-high energy neutrinos or protons/nuclei from unknown sources.

\subsection{COHERENT NEUTRINO ELASTIC SCATTERING}

In this category, there are two possible methods: use of order $G_{F}$ effect and of $G_{F}^{2}$ effect where $G_{F}$ is the Fermi constant.

\subsubsection{Order $G_{F}$ effect}

Coherent elastic scattering occurs when the de Broglie wavelength of $\mathrm{C} v \mathrm{~B} h / p_{v} \sim h / 4 T_{v} \sim 2.4 \mathrm{~mm}$ for relativistic and unclustered neutrinos, or $1.2 \mathrm{~mm} \cdot 1 \mathrm{eV} / m_{v}(\mathrm{eV})$ for clustered non-relativistic 
neutrinos is much larger than inter-atomic spacing of the target material. When a neutrino with momentum $p$ goes through coherent elastic scattering on the target and emerging with momentum $p^{\prime}$, a concept of neutrino optics can be introduced with an index of refraction $n=p^{\prime} / p$ and $n-1 \sim G_{F}$ [5]. Uses of neutrino optics were proposed to detect $\mathrm{C} v \mathrm{~B}$ either through refraction [13] or through total reflection [14] as an order $G_{F}$ effect. However, it was pointed out that the force induced by a linear momentum or energy exchange by neutrinos is canceled out to order $G_{F}$ when the target is in a uniform neutrino density $[15,16]$.

The only $G_{F}$ effect viable for detection of $\mathrm{C} v \mathrm{~B}$ is the method proposed by Stodolsky that uses an energy split of the two spin states of non-relativistic electrons in the target immersed in $\mathrm{C} v \mathrm{~B}$ for which polarized electrons in the target are needed [17]. However, this energy split is proportional to $n_{v}-n_{\bar{v}}[10,16]$. Following Duda et al. here only their result in an optimistic case of a very large asymmetry favouring $v$ with $n_{v}-n_{\bar{v}} \approx n_{v}$ is presented. Note that for overdensity of $20\left(m_{v}=0.6 \mathrm{eV}\right)$ and of $1.4\left(m_{v}=0.15 \mathrm{eV}\right)$ as estimated by Ringwald et al., $n_{v}-n_{\bar{v}}=0.95 n_{v}$ and $0.28 n_{v}$, respectively. In this approximation the authors find the energy splits for relativistic (R)/non-relativistic (NR), clustering (C)/non-clustering (NC) and Dirac (D)/Majorana (M) neutrinos: $(\Delta E)_{R}^{D}=(\Delta E)_{R}^{M}=$ $2 \sqrt{2} g_{A} G_{F}\left|\beta_{\text {Earth }}\right| n_{v}$, where $\left|\beta_{\text {Earth }}\right|=<\left|\beta_{v}\right|>$ is the velocity of the Earth relative to $\mathrm{C} v \mathrm{~B}$ normalized to the speed of light in vacuum $c$, and $g_{A}$ is $1 / 2$ for $v_{e}$ and $-1 / 2$ for $v_{\mu, \tau}$. According to Ringwald et al. $<\beta_{v}>=1.4 \times 10^{-3}$ and $2.2 \times 10^{-3}$ for overdensity of 20 and 1.4, respectively. For non-clustered (NC) non-relativistic neutrinos (NR), $(\Delta E)_{N C, N R}^{M}=2(\Delta E)_{N C, N R}^{D} \simeq$ $1.7 \sqrt{m_{v} /\left(1.7 \times 10^{-4} \xi \mathrm{eV}\right)}(\Delta E)_{R}^{D}$, and for clustered $(\mathrm{C})$ neutrino non-relativistic (NR) neutrinos $(\Delta E)_{C, N R}^{D}=\sqrt{2} g_{A} G_{F}\left|\beta_{\text {Earth }}\right| n_{v}$ and $(\Delta E)_{C, N R}^{M} \simeq 0$. In the case of Dirac relativistic neutrinos the difference in energy $\Delta E$ between the two helicity states of an electron in the direction of $\mathrm{C} \nu \mathrm{B}$ induces a torque of magnitude $\Delta E / \pi$. For a target of mass number $A$, mass $M$, and linear dimension $R$ with its moment of inertia $I=M R^{2} / \gamma$ ( $\gamma$ is a geometric factor), the acceleration due to $\mathrm{C} v \mathrm{~B}$ is $a=$ $10^{-27} f(\gamma / 10)(100 / A)(1 \mathrm{~cm} / R)\left(\beta_{\text {Earth }} / 10^{-3}\right) \mathrm{cm} / \mathrm{s}^{2}$ where $f$ is neutrino overdensity $n_{v} /<n_{v}>$ [10]. The current measurable acceleration using Cavendish type torsion balance mechanism is $\sim 10^{-13} \mathrm{~cm} / \mathrm{s}^{2}$ [18]. With possible improvements with the current technology a sensitivity down to $10^{-23} \mathrm{~cm} / \mathrm{s}^{2}$ may be achieved $[11,18,19]$. With an optimistic neutrino overdensity of $10, \gamma=10, A=100$, and $R=1 \mathrm{~cm}$, the expected acceleration is $10^{-26} \mathrm{~cm} / \mathrm{s}^{2}$ to be compared with the most optimistic sensitivity to the smallest detectable acceleration of $10^{-23} \mathrm{~cm} / \mathrm{s}^{2}$. Even in this optimistic situation, the target mass and size would need to be increased by a factor of 1000 .

\subsubsection{Order $G_{F}^{2}$ effect}

When the Earth moves through the sea of $\mathrm{C} v \mathrm{~B}$ neutrinos, a target on Earth experiences, by elastic scattering, momentum transfer from neutrinos. In the Earth's rest frame (the laboratory frame) the momentum transfer per scattering is: $<\Delta p>_{R} \approx \beta_{\text {Earth }}\left(E_{v} / c\right)$ for relativistic neutrinos, < $\Delta p>_{N C, N R}=\beta_{\text {Earth }}\left(4 T_{v} / c\right)=<\Delta p>_{R}$ for non-clustering (NC) non-relativistic (NR) neutrinos, and $<\Delta p>_{C, N R} \approx \beta_{\text {Earth }} \mathrm{cm}_{v}$ for clustered (C) non-relativistic (NR) neutrinos. The acceleration by $\mathrm{C} v \mathrm{~B}$ is given by $a=\Phi_{\nu}\left(N_{A v} / A\right) \sigma_{v-A}<\Delta p>$ where $\Phi_{v}$ is the CvB flux, A is the mass number of the target, $N_{A v}$ is the Avogadro number, and $\sigma_{v-A}$ is the neutrino-nucleus cross section. $\sigma_{v-A}=G_{F}^{2} m_{v}^{2} / \pi \approx 10^{-56}\left(m_{v} / \mathrm{eV}\right)^{2} \mathrm{~cm}^{2}$ for NR neutrinos and $=G_{F}^{2} E_{v}^{2} / \pi \approx 5 \times 10^{-63}\left(T_{v} / 1.9 \mathrm{~K}\right) \mathrm{cm}^{2}$ for $\mathrm{R}$ neutrinos. When the target satisfies the aforementioned condition for coherent elastic scattering, the cross section gets a coherent enhancement factor $A^{2}$. Then the acceleration is: $a=$ $\left(N_{A v} / A\right) n_{v}\left(G_{F}^{2} / \pi\right) A^{2} F$ where $F=\left(4 T_{v}\right)^{3} \beta_{\text {Earth }}$ for R neutrinos, $F=m_{v}^{2}\left(4 T_{v}\right) \beta_{\text {Earth }}$ for NC-NR neutrinos, and $F=m_{v}^{3} v_{v}^{2}$ for $\mathrm{C}-\mathrm{NR}$ neutrinos [10]. This result includes the nuclear coherent enhancement factor $A^{2}$ but does not include another possible coherent enhancement factor $N_{c}$ due to the larger de Broglie wavelength of $\mathrm{C \nu B}$ than the target size where $N_{c}=$ $\left(N_{A v} / A\right) \rho\left(\lambda_{v}\right)^{3}$ with $\rho$ being the density of the target $[11,20,21]$. With this enhancement, Duda et al. found that the acceleration due to $\mathrm{C} v \mathrm{~B} a=2 \times 10^{-34} f \rho\left(\mathrm{g} \mathrm{cm}^{3}\right) \mathrm{cm} / \mathrm{s}^{2}$ for R Dirac/Majorana neutrinos, $3 \times 10^{-28} f\left(m_{v}(\mathrm{eV})\right)^{2}\left(T_{v} / 1.9 \mathrm{~K}\right)^{-2} \rho\left(\mathrm{g} \mathrm{cm}^{3}\right) \mathrm{cm} / \mathrm{s}^{2}$ for NC-NR Dirac neutrinos, and $10^{-27} f \rho\left(\mathrm{g} \mathrm{cm}^{3}\right) \mathrm{cm} / \mathrm{s}^{2}$ for C-NR Dirac neutrinos. Note that for NR Majorana neutrinos, the corresponding cross sections are reduced by $\beta_{v}^{2} \approx 10^{-6}$. Even with an optimistic scenario of $f \rho \sim 100, a \approx 10^{-25} \mathrm{~cm} / \mathrm{s}^{2}$ is too small compared with the aforementioned smallest detectable acceleration of $10^{-23} \mathrm{~cm} / \mathrm{s}^{2}$.

\subsubsection{Experimental status of coherent elastic neutrino nucleus scattering (CENNS)}

As described above, the methods proposed in this category relies on the big enhancement factor due to CENNS. Although CENNS has been known since the paper by D. Freedman [22], no observation of this reaction has been made. Recently CENNS attracted close attention and several proposals have been made. Wong et al. and Barbeau et al. propose to use antineutrinos from nuclear reactors $[23,24]$. The proposal by Akimov et al. plans to use neutrinos from Stopped-pion Spallation Neutron Source (SSNS) at Oakridge National Laboratory, while Brice et al. propose to utilise neutrinos at the Fermilab Booster Neutrino Beam (BNB) [25, 26].

\subsection{NEUTRINO CAPTURE BY CvB-DECAYING NUCLEI}

The first suggestion to use neutrino capture by $\beta$-decaying nuclei (NCB) was by Weinberg in 1962 to detect $\mathrm{C} v \mathrm{~B}$ [27]. Electron (anti)neutrino capture by a nucleus $N$ that naturally undergoes beta (positron) decay to the daughter nucleus $N^{\prime}$ as $v_{e} / \bar{v}_{e}+$ $N \rightarrow N^{\prime}+e^{+} / e^{-}$has no energy threshold on the incident (anti)neutrino energy. In $\beta$-decay in which the energy conservation requires $M(N)-M\left(N^{\prime}\right)=Q_{\beta}>0$ where $M(N)$ and $M\left(N^{\prime}\right)$ are the masses of nucleus of $N$ and $N^{\prime}$, respectively, and $Q_{\beta}$ is the kinetic energy of electron/positron. For massive neutrino of mass $m_{v}$, the electron kinetic energy in NCB is $E_{e}=Q_{\beta}+E_{v} \geq Q_{\beta}+$ $m_{v}$, while, neglecting the nucleus recoil energy, $E_{e} \leq Q_{\beta}-m_{v}$ for electrons from $\beta$-decay. Thus there is a gap of $2 m_{v}$ around $Q_{\beta}$ in electron kinetic energy between $\beta$-decay and neutrino capture event. Cocco et al. find that the NCB cross section times neutrino velocity can be written as $\sigma_{N C B} v_{v}=2 \pi^{2} \ln 2 /\left(A t_{1 / 2}\right)$ where $A$ is a function of $E_{v}$ only, when the target nucleus characterized by $Q_{\beta}$ 
and $Z$ is given, and $t_{1 / 2}$ is the half-life of the target nucleus [28]. As $\beta$-decay events are the major background source to the NCB, larger ratio of the NCB to $\beta$-decay events $\sim \sigma_{N C B}\left(v_{\nu} / c\right) t_{1 / 2}$ is preferable. Among $\beta$-decaying nuclei isotopes ${ }^{3} \mathrm{H}$ and ${ }^{187} \mathrm{Re}$ have the large ratios $3.0 \times 10^{5}$ and $5.9 \times 10^{7}$, respectively. However, the fact that $\sigma_{N C B}\left(v_{v} / c\right)$ for ${ }^{3} \mathrm{H}$ and ${ }^{187} \operatorname{Re}$ are $7.8 \times 10^{-45} \mathrm{~cm}^{2}$ and $4.3 \times 10^{-52} \mathrm{~cm}^{2}$, respectively, ${ }^{3} \mathrm{H}$ seems the best choice. Although there is a gap of $2 m_{v}$ in the electron kinetic energy between the endpoint of the $\beta$-decay and the NCB events, the success of this method depends on the energy resolution whose effect may fill this gap. Cocco et al. estimate the signal (NCB) to the background ( $\beta$-decay) event ratio $R$ in the outgoing electron kinetic energy region $W_{0}-\Delta<E_{e}<W_{0}$ where $\Delta$ is the energy resolution and $W_{0}$ is the endpoint energy. They find that for $R=3$ and $m_{v}=0.7(0.3) \mathrm{eV}$ the energy resolution should be better than $0.2(0.1) \mathrm{eV}$. The event rate per mole per year is calculated to be $2.85 \times 10^{-2}\left[\sigma_{N C B}\left(v_{v} / c\right) / 10^{-45}\right] \mathrm{cm}^{2} \mathrm{yr}^{-1} \mathrm{~mol}^{-1}$. With $100 \mathrm{~g}$ of ${ }^{3} \mathrm{H}$ as the target, the event rate per year is, for $m_{v}=0.6 \mathrm{eV}, 7.5$, 90, and 150 events using the standard Fermi-Dirac (FD) distribution, Navarro-Frank-White profile (NFWhalo), and the present day mass distribution of the Milky Way (MWnow), respectively. For $m_{v}=0.3(0.15) \mathrm{eV}$, the rate is 7.5 (7.5), 23 (10), and 23 (12), respectively, with FD, NFWhalo and MWnow distribution [11].

A calculation by Faessler et al. [29] finds that the NCB rate per year for the ${ }^{3} \mathrm{H}$ target $(50 \mu \mathrm{g})$ to be used for the KATRIN experiment [30] is $4.2 \times 10^{-6} n_{v} /<n_{v}>$ which is consistent with the result by Cocco et al. above for the FD distribution with $n_{v} /<n_{v}>=1$. A similar calculation for $760 \mathrm{~g}$ of ${ }^{187} \mathrm{Re}$ to be used for the MARE calorimetric experiment [31] finds the event rate per year $6.7 \times 10^{-8} n_{v} /<n_{v}>$, which is too small for a likely value for $n_{v} /<n_{v}>$.

According to Lazauskas et al. [32], the event rate per year per Mcu (mega-curries $=3.7 \times 10^{16}$ decays $\sim 2.1 \times 10^{25}{ }^{3} \mathrm{H}$ atoms) for approximately $100 \mathrm{~g}$ of ${ }^{3} \mathrm{H}$ is $6.5 \times n_{v} /<n_{v}>\mathrm{yr}^{-1} \mathrm{Mcu}^{-1}$, which is also consistent with the result by Cocco et al. As for the required energy resolution to have a reasonable signal to noise ratio of unity, they conclude that the resolution should be a factor of two or more smaller than the neutrino mass $m_{v}$, which is similar to the conclusion by Cocco et al.

Note that the KATRIN experiment's goal for the energy resolution is $0.93 \mathrm{eV}$ and the mass of the ${ }^{3} \mathrm{H}$ source is only $50 \mu \mathrm{g}$ [30] and to increase the mass of the ${ }^{3} \mathrm{H}$ source or to improve the energy resolution it is necessary to seek a new way to perform this type of experiment. Toward this goal of improvement the Project 8 experiment has started [33]. This experiment utilizes detection of cyclotron radiation from $\beta$-decay electron to achieve an improvement in the energy resolution. Another proposal to improve the neutrino mass resolution is to use cold atomic tritium rather than molecular tritium used for the KATRIN experiment to detect both the electron and ${ }^{3} \mathrm{He}$ in the final state of $\beta$ decay [34]. Finally the PTOLEMY experiment (Princeton Tritium Observatory for Light-Early Universe Massive-neutrino Yield) is most ambitious [35]. It is based on relic neutrino capture on tritium. It uses, in addition to MAC-E Filter (Magnetic Adiabatic Collimation combined with an Electrostatic Filter) adopted in the KATRIN experiment, a large surface-deposition tritium target, cryogenic calorimeter, RF tracking similar to the Project 8 experiment, and time-of-flight systems to achieve the required background suppression and enough event rate. PTOLEMY plans to use 100 -g of atomic tritium as the target which can provide enough event rate.

\subsection{COSMIC RAYS - C $v$ B SCATTERING}

It is argued that the Universe is opaque to electrons, nucleons and photons at energies higher than $10^{23} \mathrm{eV}$ [36]. A more careful consideration finds that the interaction between cosmic ray proton and $\mathrm{CMB}$ photon $p+\gamma_{C M B} \rightarrow \pi+N$ imposes an energy threshold known as Greisen-Zatsepin-Kuzmin (GZK) cutoff $E_{G Z K} \approx 5 \times 10^{19} \mathrm{eV}$, beyond which cosmic ray protons do not survive $[37,38]$. However, Weiler argues that the only particle that can survive the GKZ cutoff is neutrinos and interactions of ultra-high energy cosmic ray neutrinos with $\mathrm{C} \nu \mathrm{B}$ through the $Z$ resonance $v+\bar{v} \rightarrow Z \rightarrow p+$ any introduces a dip at certain energy in ultra-high energy neutrino flux. If these neutrinos come from sources with the redshift $z=3.5$, this dip occurs at $9 \times$ $10^{19} \mathrm{eV}$. Depending on the three neutrino masses and the value of redshift parameter $z$ of the sources of these ultra-high energy neutrinos, there may be possibly three dips in energy spectrum of cosmic ray neutrinos if these sources exist. The existence of these neutrino sources can produce cosmic rays beyond the GZK cutoff through the $Z$ resonance (Z-burst). However, although the AGASA experiment claimed that they detected cosmic ray events above the GZK cutoff [39], neither the HiRes Experiment [40] nor the Auger experiment [41] confirmed such events.

As for detailed theoretical analyses on the $\mathrm{C} v \mathrm{~B}$ spectroscopy using ultra-high energy cosmic rays, refer to the papers by Barenboim et al. [42] and by D'Olivo et al. [43]. A detailed theoretical analysis on the Z-burst can be found, for example, in the paper by Fargion et al. [44].

Another interaction of cosmic rays with $\mathrm{C} \nu \mathrm{B}$ to detect $\mathrm{C} \nu \mathrm{B}$ was proposed by Wigman [45]. He proposes to explain, in terms of inverse $\beta$-decay interactions such as $p+\bar{v}_{e} \rightarrow n+e^{+}$, the changes in the power index of cosmic rays spectrum around $10^{15.3} \mathrm{eV}$ (the first knee) observed by the CASA-BLANCA experiment [46] from $n=2.72 \pm 0.02$ to $n=2.95 \pm 0.02$, and also at the second knee around $10^{17.5} \mathrm{eV}$ from $n=3.01 \pm 0.06$ to $n=3.27 \pm 0.02$ observed by the Fly's Eye experiment [47]. In the interaction $p+\bar{v}_{e} \rightarrow n+e^{+}$where $\bar{v}_{e}$ is $\mathrm{Cv} \mathrm{B}$, the center of mass energy $E_{C M} \approx \sqrt{m_{p}^{2}+2 E_{p} m_{v}}$ should be greater than the sum of proton and neutron masses $m_{p}+m_{n}$. This leads to the threshold proton energy for the interaction at $1.695 \times$ $10^{15} /\left(m_{v} /(1 \mathrm{eV})\right) \mathrm{eV}$. If the energy of the knee is, combining several experimental data, at $(3 \pm 1) \times 10^{15} \mathrm{eV}$ and the cause of the first knee is the inverse $\beta$-decay interaction by proton, it is consistent with the neutrino mass $m_{v_{e}}=0.5 \pm 0.2 \mathrm{eV} / \mathrm{c}^{2}$.

Although evidence of high energy cosmic ray neutrinos has finally emerged as the IceCube experiment shows [48], there is only one experimental result that suggests that there are cosmic rays beyond the GZK cutoff, which has not been confirmed by the HiRes and Auger experiment. Various mechanisms in addition to the inverse $\beta$-decay theory have been proposed to explain the existence of the knees in cosmic ray spectrum [49]. However, it is not clear which explanation is the correct model for the knees. 


\section{FUTURE PROSPECT AND CONCLUSION}

In a half-century history of studies on detection of $\mathrm{C} v \mathrm{~B}$, interesting proposals have been presented. However, with the current available technology, none of the proposed methods that are described in this review are close to be reality, except for the promising Project 8 and PTOLEMY experiment, unless local neutrino overdensity is much larger than expected. For the method to measure the acceleration due to momentum transfer by $\mathrm{C} v \mathrm{~B}$ using torsion balance, the sensitivity of detector needs to be improved by a factor of 1000 , even under optimistic circumstances. In addition the contribution from fake signal from various background sources should be carefully evaluated. Detecting $\mathrm{C} v \mathrm{~B}$ by analyzing cosmic ray spectrum as dips beyond the GZK cutoff needs a much larger cosmic ray detector, even if Nature is kind enough to provide ultra-high energy neutrino sources. The existence of the knee-like structures may be explained by other mechanisms than the inverse $\beta$-decay. Among $\beta$-decaying nuclei as the target, tritium ${ }^{3} \mathrm{H}$ seems to provide the best chance for an NCB experiment to eventually detect $\mathrm{C} v \mathrm{~B}$ if the ideas of the Project 8 and PTOLEMY experiment work to bring better energy resolution and if $\sim 100$-g of ${ }^{3} \mathrm{H}$ source, especially, of atomic tritium can be manufactured. However, this method cannot detect $v_{\mu, \tau} \mathrm{s}$.

Note added After the submission of this manuscript, a paper by Safdi et al. appeared [50]. This paper describes annual modulation of $\mathrm{C} v \mathrm{~B}$ local density caused by gravitational focusing by the Sun. This modulation can serve as a diagnostic for the signal due to $\mathrm{C} \nu \mathrm{B}$ for an experiment based on neutrino capture by $\beta$-decaying nuclei.

\section{ACKNOWLEDGMENT}

This work is in part supported by PSC-CUNY Research Award 66111-00 44.

\section{REFERENCES}

1. Kolb E, Turner M. The Early Universe. Boulder: Westview Press (1994).

2. Weinberg S. Cosmology. New York, NY: Oxford University Press (2007).

3. Bergstroem L, Goobar A. Cosmology and Particle Astrophysics. New York, NY: John Wiley and Sons (1999).

4. Sarkar S. Big bang nucleosynthesis and physics beyond the standard model Rep Prog Phys. (1996) 59:1493. doi: 10.1088/0034-4885/59/12/001

5. Gelmini GB. Prospect for relic neutrino searches. In: Proceedings of Nobel Symposium 129 (Enkoping, Sweden) (2005) arXiv:hep-ph/ 0412305 .

6. Castorina E, Franca U, Lattanzi M, Lesgourgues J, Mangano G, Melchiorri A, et al. Cosmological lepton asymmetry with a nonzero mixing angle $\theta_{13}$. Phys Rev. (2012) D86:023517. doi: 10.1103/PhysRevD.86.023517

7. Beringer J, Arguin JF, Barette RM, Copic K, Dahl O, Groom DE, et al. Review of particle physics. Phys Rev. (2012) D86:010001 (2013 update for the 2014 edition available online at: http://pdg.lbl.gov).

8. Ade PAR, Aghanim N, Armitage-Caplan C, Arnaud M, Ashdown M, AtrioBarandela F, et al. PLANCK 2013 Results. XVI. Cosmological Parameters. (2013) arXiv: 13035076.

9. Giusarma E, Di Valentino E, Lattanzi M, Michiorri A, Mena O. Relic Neutrinos, Thermal Axions and Cosmology in Early 2014. (2014) arXiv:14034852.

10. Duda G, Gelmini G, Nussinov S. Expected signals in relic neutrino detectors. Phys Rev. (2001) D64:122001. doi: 10.1103/PhysRevD.64.122001

11. Ringwald A, Wong YYY. Gravitational clustering of relic neutrinos and implications for their detection. JCAP. (2004) 0412:005. doi: 10.1088/1475$7516 / 2004 / 12 / 005$

12. Navarro JF, Frank CS, White SDM. The structure of cold dark matter halos. Astrophys J. (1996) 462:563. doi: 10.1086/177173
13. Opher R. Coherent scattering of cosmic neutrinos. Astron Astrophys. (1974) 37:135.

14. Lewis RR. Coherent detector for low-energy neutrinos. Phys Rev. (1980) D21:663.

15. Cabbibo N, Maiani L. The vanishing of order-G mechanical effects of cosmic massive neutrinos on bulk matter. Phys Lett. (1982) B114:115.

16. Langacker P, Leveille JP, Shaiman J. On the detection of cosmological neutrinos by coherent scattering. Phys Rev. (1983) D27:1228.

17. Stodolsky L. Speculation on detection of the neutrino sea. Phys Rev Lett. (1974) 34:110. doi: 10.1103/PhysRevLett.34.110

18. Hagmann C. Cosmic neutrinos and their detection. In: Proceedings of DPF 99 Meeting (Los Angeles CA) (1999) arXiv:astro-ph/9905258 .

19. Hagmann C. A relic neutrino detector. In: Proceedings of the Conference on Particle Physics and the Early Universe. AIP conference proceedings, Vol. 478 (Asilomar, CA) (1998) arXiv:astro-ph/9902102.

20. Zel'dovich YB, Khlopov MY. The neutrino mass in elementary-particle physics and in big bang cosmology. Sov Phys Usp. (1981) 24:755. doi: 10.1070/PU1981v024n09ABEH004816

21. Smith P, Lewin J. Coherent interaction of galactic neutrinos with material targets. Phys Lett. (1983) 127B:185.

22. Freedman D. Coherent effects of a weak neutral current. Phys Rev. (1974) D9:1389.

23. Wong HT, Li HB, Li J, Yue Q, Zhou ZY. Research program towards observation of neutrino-nucleus coherent scattering. J Phys Conf Ser. (2006) 39:266. doi: 10.1088/1742-6596/39/1/064

24. Barbeau PS, Collar JI, Tench O. Large-mass ultra-low noise germanium detectors: performance and applications in neutrino and astroparticle physics. JCAP. (2007) 09:004. doi: 10.1088/1475-7516/2007/09/009

25. Akimov D, Bernstein A, Barbeau P, Barton P, Bolozdynya A, Cabrera-Palmer $\mathrm{B}$, et al. Coherent Scattering Investigations at the Spallation Neutron Source: a Snowmass White Paper. (2013) arXiv:13100125.

26. Brice SJ, Cooper RL, DeJongh F, Engel A, Garrison LM, Hime A, et al. A method for measuring coherent elastic neutrino-nucleus scattering at a far off-axis high-energy neutrino beam target. Phys Rev. (2014) D89: 072004 .

27. Weinberg S. Universal neutrino degeneracy. Phys Rev. (1962) 128:1457. doi: 10.1103/PhysRev.128.1457

28. Cocco AG, Mangano G, Messina M. Probing low energy neutrino backgrounds with neutrino capture on beta decaying nuclei. JCAP. (2007) 0706:015. doi: 10.1088/1742-6596/110/8/082014

29. Faessler A, Hodak R, Kovalenko S, Simkovic F. Beta decaying nuclei as a probe of cosmic neutrino background. (2011) arXiv:11021799.

30. Parno DS. The KATRIN experiment: status and outlook. In: Proceedings of the Sixth Meeting on CPT and Lorentz Symmetry (Bloomington, IN) (2013) arXiv: 1307.5289

31. Nucciotti A. The MARE project. J Low Temp Phys. (2008) 151:597. doi: 10.1007/s10909-008-9718-5

32. Lazauskas R, Vogel P, Volpe C. Charged current cross section for massive cosmological neutrino impinging on radioactive nuclei. J Phys. (2008) G35:025001. doi: 10.1088/0954-3899/35/2/025001

33. Oblath NS. Project 8: using radio-frequency techniques to measure neutrino mass. In: Proceedings of DPF 2013 (Santa Cruz, CA) (2013) arXiv: 1310.0397.

34. Jerkins M, Klein JR, Majors JH, Robicheaux F, Raizen MG. Using cold atoms to measure neutrino mass. New J Phys. (2010) 12:043022. doi: 10.1088/13672630/12/4/043022

35. Betts S, Blanchard WR, Carnevale RH, Chang C, Chens C, Chidzik S. Development of a relic neutrino detection experiment at PTOLEMY. (2013) arXiv:13074738.

36. Weiler T. Resonant absorption of cosmic ray neutrinos by the relic neutrino background. Phys Rev Lett. (1982) 49:234. doi: 10.1103/PhysRevLett.49.234

37. Greisen K. End to the cosmic-ray spectrum? Phys Rev Lett. (1966) 16:748. doi: 10.1103/PhysRevLett.16.748

38. Zatsepin GT, Kuzmin VA. Upper limit of the spectrum of cosmic rays. JETP Lett. (1966) 4:78.

39. Takeda M, Hayashida N, Honda K, Inoue N, Kadota K, Kakimoto F, et al. Extension of the cosmic-ray energy spectrum beyond the predicted Greisen-Zatsepin-Kuzmin cutoff. Phys Rev Lett. (1998) 81:1163. doi: 10.1103/PhysRevLett.81.1163 
40. Sokolsky P. Final results from the high resolution Fly's Eye (HiRes) experiment. Nucl Phys B. (2013) 212-213:74.

41. Abraham J, Abreu P, Aglietta M, Ahn EJ, Allard D, Allen J, et al. Measurement of the energy spectrum of cosmic rays above $10^{18} \mathrm{eV}$ using the Pierre Auger observatory. Phys Lett. (2010) B685:239. doi: 10.1016/j.physletb.2010.02.013

42. Barenboim G, Requejo OM, Quigg C. Diagnostic potential of cosmicneutrino absorption spectroscopy. Phys Rev. (2005) D71:083002. doi: 10.1103/PhysRevD.71.083002

43. D’Olivo JC, Nellen L, Sahu S, Van Elewyck V. UHE neutrino damping in a thermal gas of relic neutrinos. Astropart Phys. (2006) 25:47. doi: 10.1016/j.astropartphys.2005.11.005

44. Fargion D, Mele B, Salis A. Ultra-high-energy neutrino scattering onto relic light neutrinos in the galactic halo as a possible source of the highest energy extragalactic cosmic rays. Astrophys J. (1999) 517:725. doi: 10.1086/307203

45. Wigman R. PeV cosmic rays: window on the leptonic era? Astropart Phys. (2003) 19:379. doi: 10.1016/S0927-6505(02)00242-6

46. Fowler JW, Fortson LF, Jui CCH, Kieda DB, Ong RA, Pryke CL, et al. A measurement of the cosmic ray spectrum and composition at the knee. Astropart Phys. (2001) 15:49. doi: 10.1016/S0927-6505(00)00139-0

47. Watson AA. The highest energy cosmic rays. Nucl Phys B. (1991) 22:116. doi: 10.1016/0920-5632(91)90011-3

48. Artsen MG, Abbasi R, Abdou Y, Ackermann M, Adams J, Aguilar JA, et al. Evidence for high-energy extraterrestrialneutrinos at the
IceCube detector. Science (2013) 342:1242856. doi: 10.1126/science. 1242856

49. Bluemer J, Engel R, Hoerandel JR. Cosmic rays from the knee to the highest energies. Prog Part Nucl Phys. (2009) 63:293-338. doi: 10.1016/j.ppnp.2009.05.002

50. Safdi BR, Lisanti M, Spitz J, Formaggio JA. Annual modulation of cosmic relic neutrinos. (2014) arXiv:14040680.

Conflict of Interest Statement: The author declares that the research was conducted in the absence of any commercial or financial relationships that could be construed as a potential conflict of interest.

Received: 17 March 2014; accepted: 07 May 2014; published online: 10 June 2014. Citation: Yanagisawa C (2014) Looking for cosmic neutrino background. Front. Physics 2:30. doi: 10.3389/fphy.2014.00030

This article was submitted to High-Energy and Astroparticle Physics, a section of the journal Frontiers in Physics.

Copyright (c) 2014 Yanagisawa. This is an open-access article distributed under the terms of the Creative Commons Attribution License (CC BY). The use, distribution or reproduction in other forums is permitted, provided the original author(s) or licensor are credited and that the original publication in this journal is cited, in accordance with accepted academic practice. No use, distribution or reproduction is permitted which does not comply with these terms. 